\title{
Influence of the cognitive aspects of caregivers in the adhesion of treatment in children and adolescents with chronic rheumatic diseases-preliminary data
}

\author{
Lívia Keppeke, Claudio A Len", Vanessa Miotto e Silva, Juliana Molina, Fernanda Lewinsky, Maria Teresa Terreri
}

From 21st European Pediatric Rheumatology (PReS) Congress

Belgrade, Serbia. 17-21 September 2014

\section{Introduction}

The adhesion to treatment is a key factor for an effective treatment. Studies have showed considerable taxes in poor adhesion in children and adolescents with rheumatic diseases, which lead to long-term costs and consequences for the patient, the family, and society. Psychological conditions and familial support have demonstrated to be a strong influence in the adhesion to treatment.

\section{Objectives}

To study the psychocognitive and social aspects of the caregivers of patients with rheumatic diseases and evaluate its correlation with adhesion to treatment.

\section{Methods}

42 caregivers of patients followed in our outpatient pediatric rheumatology clinic participated in this study, classified according to good or bad adhesion to treatment, according to the Morisky Green drug adhesion test. The sample was selected consecutively. We used a standard questionnaire to verify the socioeconomic level, the Family Apgar scale to verify family functioning, The Wechsler Adult Intelligence Scale (WAIS) to detect IQ, and clinical and demographic data.

\section{Results}

We observed good adherence in $6 \%$ of patients and bad adherence in $31 \%$. In relation to socioeconomic class, $59 \%(n=17)$ of adherent patients belonged in the low middle class, against $77 \%(\mathrm{n}=10)$ of non-adherent patients $(\mathrm{p}=0.31)$. The family functioning showed itself

Pediatrics, UNIFESP, São Paulo, Brazil 